\title{
Mathematical Modeling of Diffusion Phenomenon in a Moderately Constricted Geometry
}

\author{
Shailesh Mishra, Narendra Kumar Verma, Shafi Ullah Siddiqui \\ Department of Mathematics, Harcourt Butler Technological Institute, Kanpur, India \\ E-mail: shailesh27sep@rediffmail.com \\ Received October 30, 2010; revised December 19, 2010; accepted December 24, 2010
}

\begin{abstract}
The incorporation of fluid flow through modelled normal and stenosed capillary-tissue exchange system has highlighted issues that may have major applications for the study of diffusion phenomenon. Results clearly demonstrate the important roles played by various physiological characteristics and diffusion variables involved in the analysis on blood flow. Assessment of the severity of the disease could be made possible through the variation of a parameter named as retention parameter. An attempt has been made to study the effects of local variation of viscosity on flow, wall-shearing stress and distribution of dissolved material in diseased artery as compared to the normal.
\end{abstract}

Keywords: Stenosis, Diffusion, Wall Shear Stress, Viscosity, Retention Parameter

\section{Introduction}

Atherosclerosis is a chronic disease which involves the build up of cholesterol and other fatty deposits within the arterial wall leading to the narrowing of the blood vessel lumen (Figure 1). This has the consequence of restricting blood flow to vital organs which can eventually lead to various clinical syndromes such as heart attacks and strokes. These events are the leading causes of death in the developed world $[1,2]$.

The early events leading to atherosclerosis occur in the space between the endothelial cells and the smooth muscle cells, a region of the blood vessel known as the subendothelium or intima. The process leading to the cholesterol deposits begins with the formation of fatty streaks. Accumulation of cholesterol within cells leads to the formation of 'foam cells' which reside in this intimal region of the vessel wall and constitute the fatty streak. At this stage no symptoms will be observed and in fact symptoms of atherosclerosis often do not reveal themselves until complications such as angina and coronary artery disease arise. The fatty streaks are often present in childhood and may not always progress to form the plaques which can be found in affected adults [3]. The disease may remain asymptomatic for many years. However, critical restriction of blood flow or thrombosis leading to total occlusion of the vessel may lead to cardiovascular events such as heart attack or stroke later in life.
While modeling blood flow in a stenosed tube, it was initially assumed that, the flow obeys Newtonian hypothesis and the flow variables have been computed by using basic Navier-Stoke's equation [4,5]. Later, the model has been extended by assuming that, it obeys nonNewtonian hypothesis and showed that under low shear rates, the model could be best described by this representation.

The papers [6-10] provide a small sample of the research on non-Newtonian effects on blood flow. Perkkio and Keskinen [11] studied the effects of the concentration on viscosity and the effects of the concentration on blood flow through a vessel with stenosis and found it an important aspect from physiological point of view. Kang and Eringen [12] have also discussed the effects of the variation of the concentration of the suspended cells of the blood. Viscosity depending on the local variation of the concentration of the suspended cells has been introduced by Tandon et al. $[13,14]$. In the present analysis an attempt has been made to study the effects of local variation of viscosity on flow, wall shearing stress and diffusion of dissolved material (nutrients) in diseased artery as compared to the normal. This work may help in early identification, diagnosis and treatment of cardiovascular disorders. Increasing values of $M$ represent growth of new cells which interns increases the viscosity. The resistance to flow $(\lambda)$ increases with the growth of stenosis and with the increasing values of the parameter $\alpha$ 


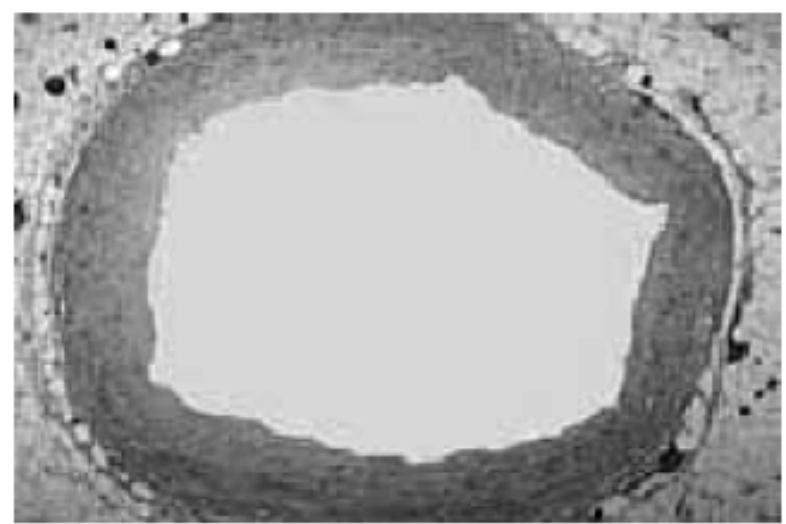

(a)

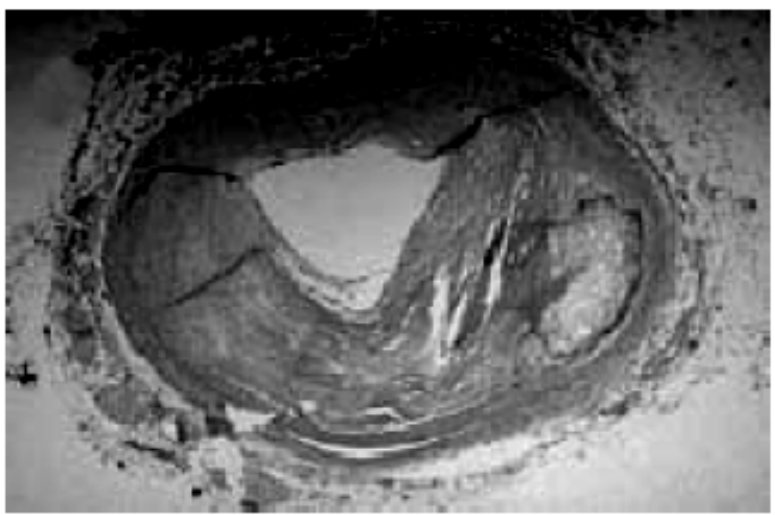

(b)

Figure 1. (a) Cross section of a normal artery lumen. The endothelial cells neighbour the lumen and no intimal thickening is present; (b) Sever atherosclerosis. On the right a calcified plaque has formed pushing against the endothelial cells them to bulge into the lumen restricting blood flow.

and $M$. Variation of wall shearing stress $\left(\tau_{s}\right)$ with the developing stenosis for different values of parameter $\alpha$ are similar as for resistance to flow. It is seen that increasing values of $N$ describe the increase in retention of the solutes within the capillary.

Consider the flow of blood through a circular tube of radius $R(z)$ whose viscosity varies along the radial direction. In the capillary segment the geometry of the stenosis is given by

$$
\frac{R(z)}{R_{0}}= \begin{cases}1-A\left[L_{0}^{m-1}(z-d)-(z-d)^{m}\right] & d \leq z \leq d+L_{o} \\ 1 & \text { otherwise }\end{cases}
$$

Here the parameter $A$ is expressed as

$$
A=\frac{\delta}{R_{0}} \frac{L_{0}^{m} m^{m /(m-1)}}{m-1}
$$

where $\delta$ denotes the maximum height of the stenosis at
$z=d+\frac{L_{0}}{m^{m /(m-1)}}$ such that $\frac{\delta}{R_{0}} \ll 1 . R(z)$ and $R_{0}$ is the radius of capillary with and without stenosis respectively (see Figure 2).

\section{Formulation of the Problem}

The flow is considered to be steady, fully developed and laminar viscous flow of suspensions of cells. For dilute suspensions, a reasonable approximation to the viscosity of the suspension may be described as

$$
\mu=\mu_{0}(1+\alpha C)
$$

where $\mu$ and $\mu_{0}$ denote the viscosity of blood and plasma respectively.

The concentration $C$ is determined by the governing diffusion equation

$$
D\left(\frac{\partial^{2} C}{\partial r^{2}}+\frac{1}{r} \frac{\partial C}{\partial r}\right)+M=0
$$

where $D$ is the diffusion coefficient and $M$ is the rate of production or degeneration.

The concentration equation for the solute is expressed as

$$
\frac{\partial C_{1}}{\partial t}+u \frac{\partial C_{1}}{\partial z}=D_{1}\left(\frac{\partial^{2} C_{1}}{\partial r^{2}}+\frac{1}{r} \frac{\partial C_{1}}{\partial r}\right)
$$

where $C_{1}$ represents the concentration of the solute, $\mathrm{u}$ is the axial velocity and $D_{1}$ the diffusion coefficient for the solute under consideration in the blood.

The equations governing the flow of blood in the arterial system are given by

$$
\begin{gathered}
0=-\frac{\partial p}{\partial z}+\frac{\mu}{r} \frac{\partial}{\partial r}\left\{r \frac{\partial u}{\partial r}\right\} \\
0=-\frac{\partial p}{\partial r}
\end{gathered}
$$

To solve the above system of equations, the following boundary and matching conditions are introduced:

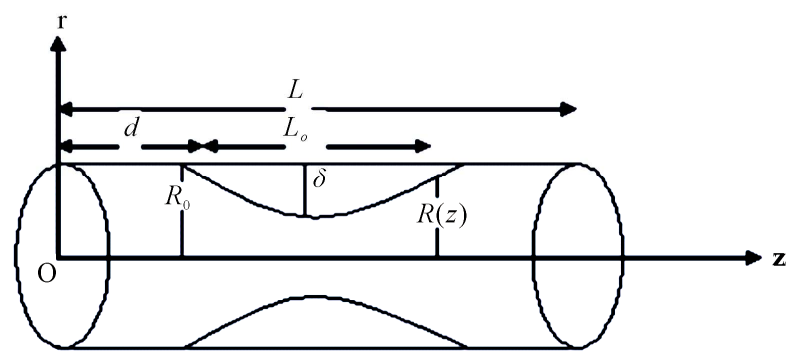

Figure 2. Flow geometry of stenosed capillary. 


$$
\begin{aligned}
& \frac{\partial C}{\partial r}=0, \quad \text { at } r=0 \\
& -D \frac{\partial C}{\partial r}=V . C, \quad \text { at } r=R \\
& \frac{\partial u}{\partial r}=0, \quad \text { at } r=0 \\
& u=0, \quad \text { at } r=R \\
& \frac{\partial C_{1}}{\partial r}=0, \quad \text { at } r=0 \\
& -D_{1} \frac{\partial C_{1}}{\partial r}=V . N . C_{1}, \quad \text { at } r=R \\
& p=p_{0}, \quad \text { at } z=0 \\
& p=p_{L}, \quad \text { at } z=L
\end{aligned}
$$

\section{Solution of the Problem}

The expression for concentration $C$ is obtained by solving Equation (4) using the boundary conditions given in Equation (8) as

$$
C=\frac{M}{4 D V}\left[V\left(R^{2}-r^{2}\right)-2 D R\right]
$$

The expression for the viscosity is given by

$$
\mu=\mu_{0}\left[A_{1} r^{2}+A_{2}\right]
$$

where $A_{1}=-\frac{\alpha M}{4 D}$ and $A_{2}=1+\frac{\alpha M}{4 D V}\left(V R^{2}-2 D R\right)$.

On solving Equation (6) with the help of boundary conditions mentioned in Equation (8) we obtained the velocity distribution

$$
u=-\frac{1}{4 A_{2} \mu_{0}}\left\{\left(R^{2}-r^{2}\right)-\frac{A_{1}}{2 A_{2}}\left(R^{4}-r^{4}\right)\right\} \frac{\partial p}{\partial z}
$$

The volumetric rate of flow is defined as

$$
Q=\pi \int_{0}^{R} r^{2}\left(-\frac{d u}{d r}\right) d r
$$

By performing the integration of Equation (12), using Equation (11), one obtains

$$
Q=-\left\{\frac{\pi}{2 A_{2} \mu_{0}}\right\}\left[\frac{R^{4}}{4}-\frac{A_{1} R^{6}}{3 A_{2}}\right] \frac{\partial p}{\partial z}
$$

The pressure gradient is thus obtained as

$$
\frac{d p}{d z}=-\frac{2 Q}{\pi I(z)}
$$

where $I(z)=\int_{0}^{R} \frac{r^{3} d r}{\mu(r)}$.

Integrating Equation (14) and using the boundary con- dition given in Equation (8), we have

$$
p_{0}-p_{L}=\frac{2 Q}{\pi} \int_{0}^{L} \frac{d z}{I(z)}
$$

The resistance to flow $\lambda$ is defined by

$$
\lambda=\frac{\left(p_{0}-p_{L}\right)}{Q}
$$

which on solving gives

$$
\lambda=\frac{2}{\pi}\left[\frac{\left(L-L_{0}\right)}{I_{0}}+\int_{d}^{d+L_{0}} \frac{d z}{I(z)}\right]
$$

where $I_{0}=\int_{0}^{R_{0}} \frac{r^{3}}{\mu(r)} d r$.

The wall shearing stress is given by

$$
\tau_{R}=\left[\mu(r) \frac{d u}{d r}\right]_{r=R(z)}
$$

which on using Equation (11) and Equation (15) gives $\tau_{s}$, given by

$$
\tau_{s}=\left[\frac{R(z) Q}{\pi I(z)}\right]
$$

Following Tandon and Pal [13] the apparent viscosity is expressed as

$$
\mu_{\text {app }}=\frac{\mu}{\mu_{0}}=\left[\frac{R^{4}}{A_{2}}-\frac{4 A_{1} R^{6}}{3 A_{2}^{2}}\right]^{-1}
$$

To solve the concentration equation for the solute given by Equation (5), following non-dimensional quantities are introduced:

$$
t_{1}=\frac{t}{\bar{t}}, \bar{t}=\frac{L}{u}, \xi=\frac{(z-u t)}{L}, \eta=\frac{r}{R_{0}}, \bar{C}_{1}=\frac{C_{1}}{C_{0}}, R^{\prime}=\frac{R}{R_{0}}
$$

so that Equation (5) becomes

$$
\left(\frac{\partial \bar{C}_{1}}{\partial t_{1}}\right) / t+\frac{v}{L} \frac{\partial \bar{C}_{1}}{\partial \xi}=\frac{\bar{D}_{1}}{R_{0}^{2}}\left[\frac{\partial^{2} \bar{C}_{1}}{\partial \eta^{2}}+\frac{\partial \bar{C}_{1}}{\partial \eta}\right]
$$

together with the boundary conditions:

$$
\frac{\partial \bar{C}_{1}}{\partial \eta}=0 \text {, at } \eta=0 ; \bar{D}_{1}\left(\frac{\partial \bar{C}_{1}}{\partial \eta}\right)=V \cdot N \cdot \bar{C}_{1} \text {, at } \eta=\frac{R}{R_{0}}
$$

where $v=u-\bar{u}, N$ is the retention parameter and

$$
\bar{u}=\frac{R_{0}^{4} \cdot R^{\prime 2}}{4 A_{2} \mu_{0} L} \frac{\partial p}{\partial \xi}
$$

and $V$ is the radial velocity of the wall, given by

$$
V=\frac{d R^{\prime}}{d t^{\prime}}
$$


If the Taylor's longitudinal condition is valid in this problem, the partial equilibrium may be assumed at any cross-section of the artery and the variation in $\bar{C}_{1}$ with $r$ is obtained from Equation (22), which may be written in the form

$$
\frac{\partial^{2} \bar{C}_{1}}{\partial \eta^{2}}+\frac{1}{\eta} \frac{\partial \bar{C}_{1}}{\partial \eta}=\frac{v R_{0}^{2}}{\bar{D}_{1} L} \frac{\partial \bar{C}_{1}}{\partial \xi}
$$

To solve Equation (25), we use boundary condition given in Equation (23) and obtain

$$
\bar{C}_{1}=\frac{R_{0}^{4}}{2 A_{2} \bar{D}_{1} L^{2} \mu_{0}} \cdot \frac{\partial p}{\partial \xi}\left[\frac{\eta^{4}}{16}-\frac{\eta^{6}}{144 R^{2}}-\frac{R^{\prime 2} \eta^{2}}{8}\right] \frac{\partial \bar{C}_{1}}{\partial \xi}+A_{3}
$$

where $A_{3}=\frac{R_{0}^{3} \cdot R^{\prime 3}}{2 A_{2} \mu_{0} L^{2}} \frac{\partial p}{\partial \xi}\left[\frac{10 V N R_{0} R^{1}}{144 \bar{D}_{1}}-1\right] \frac{\partial \overline{C_{1}}}{\partial \xi}$.

\section{Results and Discussions}

In this paper the concentration profiles and associated physiological diffusion variables involved in the analysis for normal and diseased system associated with stenosis due to the local deposition of lipids have been determined. Such models may help in identification, diagnosis and treatment of many cardiovascular disorders.

The results are shown in the Figures 3 to 9 by taking the value of parameters based on experimental data in a capillary.

$$
L_{0}(\mathrm{~cm})=1 ; L(\mathrm{~cm})=1,2,5 ; \delta / R_{0}=0.2,0.3,0.4, \cdots 0.8 ;
$$
$\alpha=0.5,1.0,1.5,2.0 ; M=1,2,3 ; m=2$ (for symmetric stenosis).

Figure 3 shows the variation of apparent viscosity $\left(\mu / \mu_{0}\right)$ with the stenosis size $\left(\delta / R_{0}\right)$ for different values of $\alpha$. It is seen that increase in $\alpha$, increases the apparent viscosity as the stenosis develops.

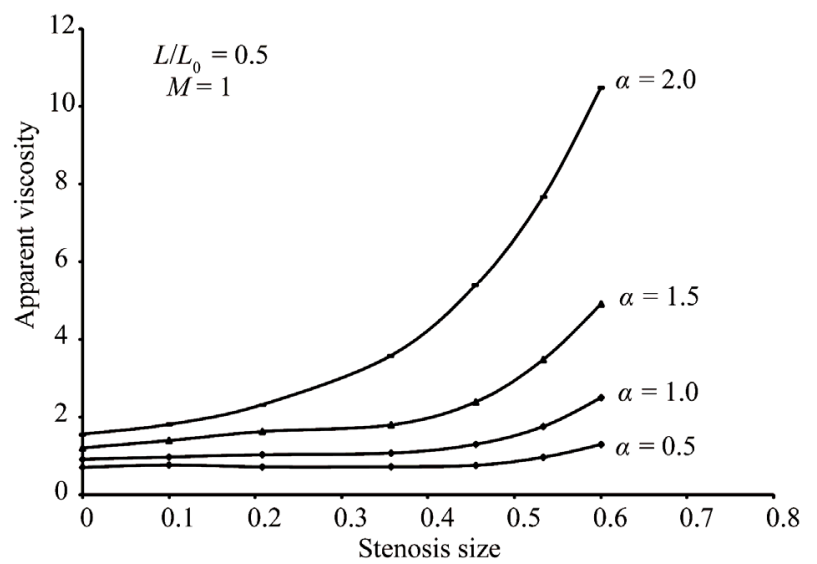

Figure 3. Variation of apparent viscosity with stenosis for different value of $\alpha$.
The effects of variations of $M$ representing the generation or degradation of the cells on apparent viscosity $\left(\mu / \mu_{0}\right)$ has been depicted in Figure 4. Increasing values of $M$ represent growth of new cells, i.e. generation. This in turn increases the viscosity. The effect of increasing values of stenosis size $\left(\delta / R_{0}\right)$ is also obvious because narrowing the arteries would increase concentration of the suspended cells owing to the flow of plasma through the stenotic region due to the growing stenosis. This effect is similar to that of collapsing walls symmetrically.

Figure 5 and Figure 6 describe the variation of the resistance to flow $(\lambda)$ with stenosis size $\left(\delta / R_{0}\right)$ for different values of parameters $\alpha$ and $M$. The resistance to flow $(\lambda)$ increases with the growth of stenosis and with the increasing values of the parameter $\alpha$ and $M$. Variation of resistance to flow with developing stenosis is similar to that obtained by Shukla et al. [15] and Mishra et al. [16].

Variation of wall shearing stress $\left(\tau_{s}\right)$ with the developing stenosis for different values of parameter $\alpha$ is presented in Figure 7. As the stenosis grows, the wall

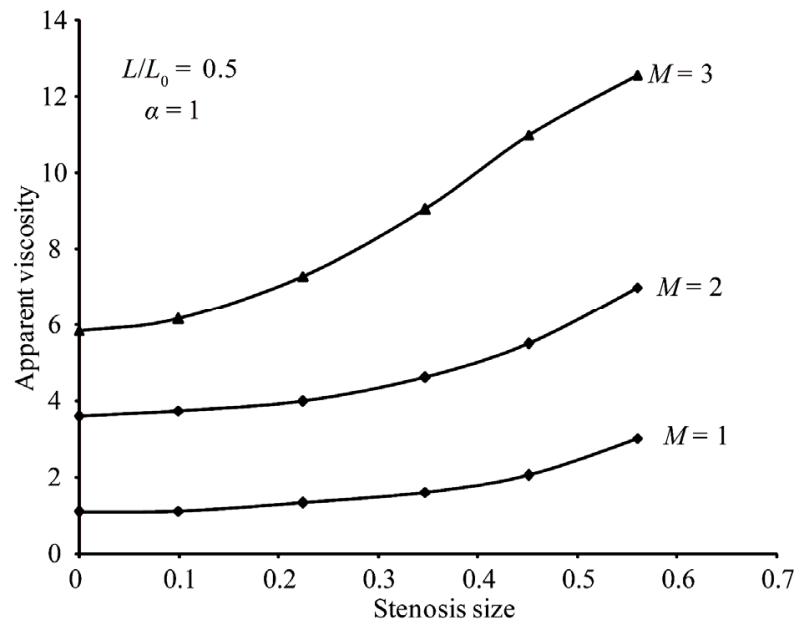

Figure 4. Variation of apparent viscosity with stenosis size for different values of $M$.

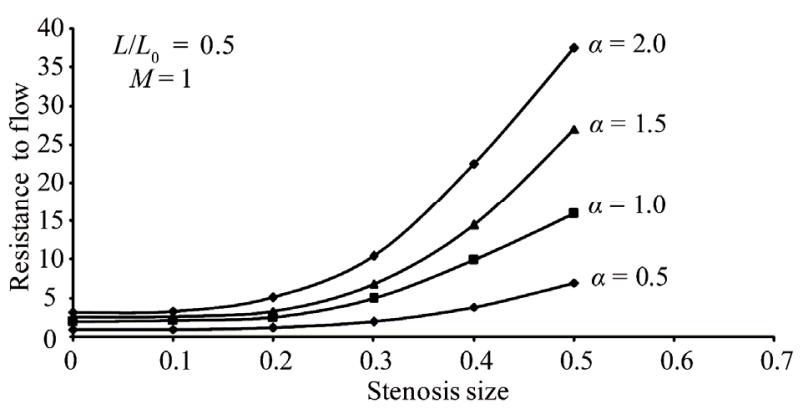

Figure 5. Variation of resistance to flow with stenosi size for different values of $\alpha$. 
shearing stress $\left(\tau_{s}\right)$ increases. The results for increasing values of $\alpha$ are similar to for resistance to flow.

Figure 8 reveals the effect of retention parameter $(N)$ on concentration in capillary. Increasing values of $N$ describe the increase in retention of the solutes within the capillary. $N=1$ implies the complete retention, i.e., no solute or fluid diffuses and as the retention parameter decreases from 1 to 0.4 more solute diffuses, which in turn, decreases the solute concentration in the capillary region. The variation of the values of retention parameter in the stenotic region may also be associated with the type of plaques deposited on the walls: calcified, fibrous or fatty plaques.

Figure 9 shows the diffusion of large and small molecular weight nutrients within the capillary for different values of stenosis size $\left(\delta / R_{0}\right)$.

Large molecular weight nutrients face more resistance to diffuse into the tissue and therefore the cells of the deeper region are deprived of getting sufficient nutrition. Similar results have been obtained by Tandon et al. [13].

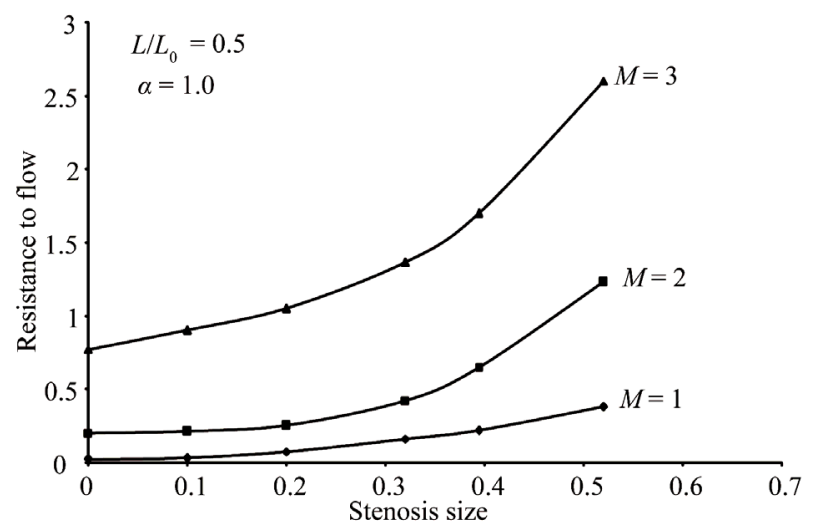

Figure 6. Variation of resistance to flow with stenosi size for different values of $M$.

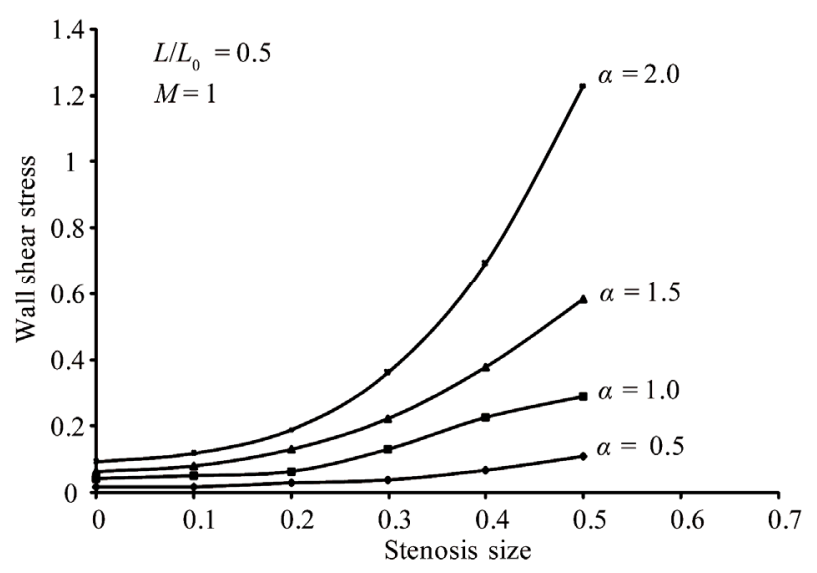

Figure 7. Variation of wall shear stress with stenosis size for different values of $\alpha$.

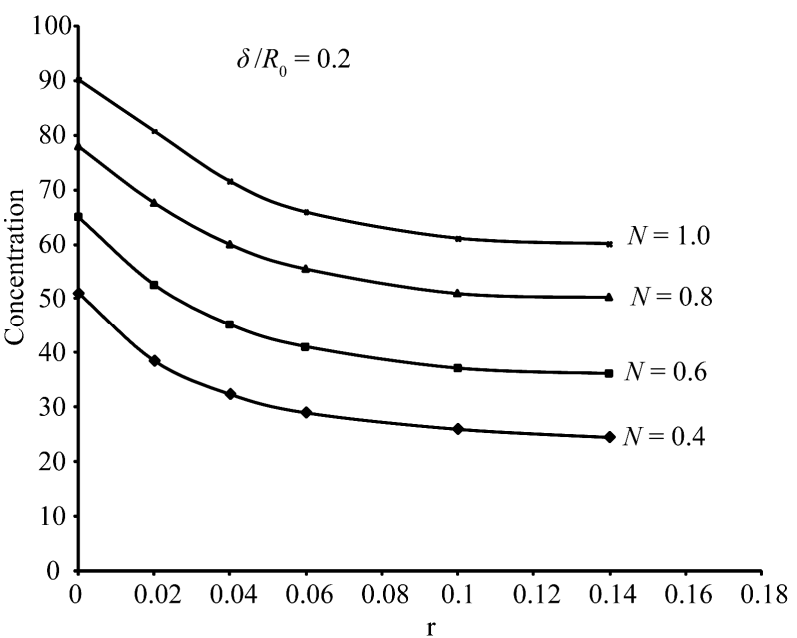

Figure 8. Concentration profiles for different values of retention parameter $(N)$.

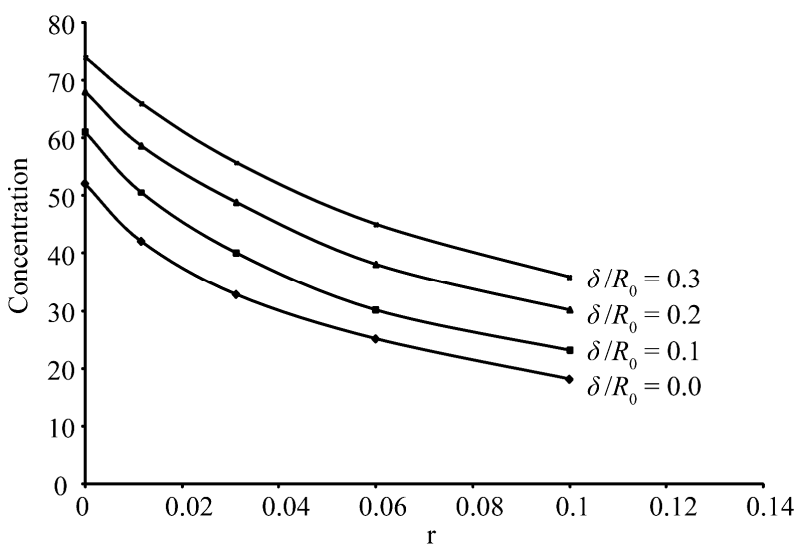

Figure 9. Concentration profiles for different values of stenosis size $\delta / R_{0}$.

\section{Concluding Remarks}

The problem relating capillary-tissue exchange phenomenon is mixed coupled boundary problem. This model has incorporated simultaneous dispersion of solute in capillary in normal and stenotic condition depending on various parameters including retention parameters. The results are more encouraging and correlating well with the experimental observation that deeper region cells are deprived of the nutrients in the stenotic region. There is a need to pursue inter-disciplinary research at a greater pace for further development from clinical point of view.

\section{References}

[1] V. W. Bowry and K. U. Ingold, "The Unexpected Role of Vitamin E ( $\alpha$-Tocopherol) in the Peroxidation of Human Low-Density Lipoprotein," Accounts of Chemical Research, Vol. 32, No. 1, 1999, pp. 27-34. 
doi:10.1021/ar950059o

[2] M. J. Davies and N. Woolf, "Atherosclerosis in Ischaemic Heart Disease: The Mechanisms,” Science Press, London, 1990.

[3] H. Esterbauer, J. Gebicki, H. Puhl and G. Jurgens, "The Role of Lipid Peroxidation and Antioxidants in Oxidative Modification of LDL," Free Radical Biology and Medicine, Vol. 13, No. 4, 1992, pp. 341-390. doi:10.1016/0891-5849(92)90181-F

[4] B. V. R. Kumar and K. B. Naidu, "A Pulsatile Suspension Flow Simulation in a Stenosed Vessel," Mathematical and Computer Modeling, Vol. 23, No. 5, 1996, pp. 75-86. doi:10.1016/0895-7177(96)00013-1

[5] P. N. Tandon and U. V. S. Rana, “A New Model for Blood Flow through an Artery with Axisymmetric Stenosis," International Journal of Biomedical Computing, Vol. 38, No. 3, 1995, pp. 257-267. doi:10.1016/S0020-7101(05)80008-X

[6] F. J. H. Gijsen, F. N. van de Vosse and J. D. Janssen, "The Influence of the Non-Newtonian Properties of Blood on the Flow in Large Arteries: Steady Flow in a Carotid Bifurcation Model," Journal of Biomechanics, Vol. 32, No. 6, 1999, pp. 601-608. doi:10.1016/S0021-9290(99)00015-9

[7] B. Johnston, P. R. Johnston, S. Corney and D. Kilpatrick, "Non-Newtonian Blood Flow in Human Right Coronary Arteries: Steady State Simulations," Journal of Biomechanics, Vol. 37, No. 5, 2004, pp. 709-720. doi:10.1016/j.jbiomech.2003.09.016

[8] A. Leuprecht and K. Perktold, "Computer Simulation of Non-Newtonian Effects on Blood Flows in Large Arteries," Computer Methods in Biomechanics \& Biomedical Engineering, Vol. 4, No. 2, 2001, pp. 149-163.

\section{doi:10.1080/10255840008908002}

[9] P. Neofytou and D. Drikakis, "Non-Newtonian Flow Instability in a Channel with a Sudden Expansion,” Journal of Non-Newtonian Fluid Mechanics, Vol. 111, No. 2-3, 2003, pp. 127-150. doi:10.1016/S0377-0257(03)00041-7

[10] K. K. Yeleswarapu, "Evaluation of Continuum Models for Characterizing the Constitutive Behavior of Blood," Ph.D. Thesis, Department of Mechanical Engineering, University of Pittsburgh, Pittsburgh, 1996.

[11] J. Perkkio and R. Keskinen, "Hematocrit Reduction in Bifurcation due to Plasma Skimming," Bulletin of Mathematical Biology, Vol. 45, No. 1, 1983, pp. 41-50.

[12] C. K. Kang and A. C. Eringen, "The Effect of Microstructure on the Rheological Properties of Blood,” Bulletin of Mathematical Biology, Vol. 38, No. 2, 1976, pp. 135-159.

[13] P. N. Tandon and T. S. Pal, "On Transmural Fluid Exchange and Variation of Viscosity of Blood Flowing through Permeable Capillaries,” Medical and Life Science Engineering, Vol. 5, No. 1, 1979, pp. 18-29.

[14] P. N. Tandon and R. Agarwal, "A Study on Nutritional Transport in Synovial Joints,” International Journal of Computers and Mathematics with Application, Vol. 17, No. 7, 1989, pp. 1101-1141.

[15] J. B. Shukla, R. S. Parihar and B. R. P. Rao, "Effects of Stenosis on Non-Newtonian Flow of the Blood in an Artery,” Bulletin of Mathematical Biology, Vol. 42, No. 3, 1980, pp. 283-294.

[16] J. C. Mishra and S. Chakravarty, "Flow in Arteries in the Presence of Stenosis,” Journal of Biomechanics, Vol. 19, No. 11, 1986, pp. 907-918. doi:10.1016/0021-9290(86)90186-7 